\title{
Monitoring the Direct Thrombin Inhibitors
}

\author{
GEORGE A. FRITSMA
}

\section{LEARNING OBJECTIVES}

1. Diagram the physiology of the direct thrombin inhibitors

2. Distinguishe between argatroban and bivalirudin.

3. Provide the means for monitoring direct thrombin inhibitors.

ABBREVIATIONS: APTT or PTT - activated partial thromboplastin time; AT - antithrombin; CAD - coronary artery disease; DTI - direct thrombin inhibitor; DVT - deep venous thrombosis; ECT-ecarin clotting time; FDA - US Food and Drug Administration; HIT heparin-induced thrombocytopenia with thrombosis; INR - international normalized ratio; LMWH - low molecular weight heparin; PE - pulmonary embolism; PCI - percutaneous intervention (cardiac catheterization); PiCT - prothrombinase-induced clotting time; PT - prothrombin time; RI - reference interval; RUO research use only; TCT - thrombin clotting time; TPA tissue plasminogen activator; UFH - unfractionated heparin; VTE - venous thromboembolism.

INDEX TERMS: Anticoagulants, heparin, Coumadin, lepirudin, argatroban, bivalirudin, dabigatran, prothrombin time, activated partial thromboplastin time, atrial fibrillation, thrombosis, thromboembolic disease, coronary artery disease, heparin-induced thrombocytopenia with thrombosis, thrombin clotting time, dilute thrombin time, ecarin clotting time, prothrombinase-induced clotting time.

Clin Lab Sci 2013;26(1):54

George A. Fritsma MS MT (ASCP), The Fritsma Factor, Your Interactive Hemostasis Resource ${ }^{S M}$, Fritsma \& Fritsma LLC, Birmingham, $A L$

Address for Correspondence: George A. Fritsma MS MT (ASCP), The Fritsma Factor, Your Interactive Hemostasis Resource $^{S M}$, Fritsma \& Fritsma LLC, 153 Redwood Drive, Birmingham, $A L$ 357173, 205-655-0687, George@ fritsmafactor.com.
The direct thrombin inhibitors (DTIs), intravenous argatroban and bivalirudin and oral dabigatran, reversibly bind and inactivate free and clot-bound thrombin without activating antithrombin (AT, antithrombin III, ATIII), thereby suppressing coagulation at the final stage of the cascade. ${ }^{1}$ Argatroban or bivalirudin are substituted for unfractionated heparin (UFH) or low molecular weight heparin (LMWH) when heparin-induced thrombocytopenia with thrombosis (HIT) is suspected or confirmed, as they do not interact with the HIT antibody. ${ }^{2}$ When HIT is suspected, the risk of either venous or arterial thrombosis is $50 \%$ for 30 days after heparin withdrawal unless argatroban or bivalirudin is employed. Coumadin is contraindicated in HIT as it is prothrombotic for the first 5 days of administration. Dabigatran has been cleared for prevention of venous thromboembolic (VTE) disease in Europe and Canada and for prevention of stroke in atrial fibrillation in the US. As the first new oral anticoagulant cleared by the FDA since 1954, dabigatran is beginning to take its place in the formulary. Laboratory scientists are challenged to find clinically effective means to monitor its plasma concentration.

\section{Argatroban}

Argatroban (Novostan ${ }^{\circledR}$; GlaxoSmithKline, Research Triangle Park, NC) is a non-protein l-arginine derivative with a molecular weight of 527 Daltons. $^{3}$ Argatroban was FDA-cleared in 1997 for anticoagulation in HIT. ${ }^{4}$ Argatroban may be used for prophylaxis, treatment, and also for anticoagulation during percutaneous cardiac intervention (PCI, cardiac catheterization) for patients with HIT. ${ }^{5}$

The physician initiates the argatroban intravenous infusion at $2 \mu \mathrm{g} / \mathrm{kg} / \mathrm{min}$ or in patients with liver disease at $0.5 \mu \mathrm{g} / \mathrm{kg} / \mathrm{min}$. During PCI, a bolus of $350 \mu \mathrm{g} / \mathrm{kg}$ is administered over 3-5 minutes followed by 25 $\mu \mathrm{g} / \mathrm{kg} / \mathrm{min}$. Argatroban is cleared by the liver and excreted in stool. There is a $5 \%$ general bleeding risk and no direct antidote; however, the half-life is 51 
minutes, and argatroban clears from the blood completely in 2-4 hours except in liver disease. Argatroban therapy may require laboratory testing, particularly for detection of an overdose or in liver disease.

\section{Lepirudin, a Synthetic Analogue of Hirudin}

Lepirudin (Refludan ${ }^{\circledR}$; ZLB Behring $\mathrm{GmbH}$, Montville, N.J., and Marburg, Germany) is a recombinant 7000 Dalton protein DTI. Lepirudin is an analogue of natural hirudin, which is produced in trace amounts by the medicinal leech Hirudo medicinalis. Hirudin is secreted in leech saliva, enabling the leech to avoid clotting while ingesting its blood meal. Lepirudin is approved for intravenous anticoagulation in HIT. It binds free but not fibrin-bound thrombin, and is cleared only by the kidneys.

Lepirudin production was discontinued May 31, 2012, though existing stocks may be used until depleted. Laboratory assays may be necessary because the risk of overdose-related bleeding is $10 \%$. The dosage must be reduced in the presence of elevated serum creatinine or reduced glomerular filtration rate (GFR) and is discontinued when the GFR is less than $15 \mathrm{~mL} / \mathrm{min}$ or the creatinine exceeds $6 \mathrm{mg} / \mathrm{dL}$. Serial measurements of GFR or serum creatinine are necessary throughout lepirudin therapy. There is no antidote for lepirudin overdose, but the plasma half-life is only 20 minutes, provided kidney function is normal.

Anti-hirudin antibodies form in about $40 \%$ of HIT patients who are treated with lepirudin. ${ }^{6}$ These antibodies complex with lepirudin and may actually increase lepirudin's anticoagulant effect, because the bound DTI remains active and the complex clears slowly. Strict laboratory monitoring is necessary during prolonged therapy, and anaphylaxis may occur during a second administration, thus lepirudin may only be used once. $^{7}$

\section{Bivalirudin, a Depolymerized Lepirudin Derivative}

Bivalirudin (Angiomax ${ }^{\circledR}$; The Medicines Company, Parsippany, NJ) is a synthetic 20-amino acid peptide derivative of lepirudin with a molecular weight of 2180 Daltons. Bivalirudin inactivates both free and clotbound thrombin. Bivalirudin was FDA-cleared in 2000 for use as an anticoagulant in patients with unstable angina at risk for HIT who are undergoing PCI. ${ }^{8}$
Bivalirudin is intended for use with concurrent aspirin therapy at a dosage of $325 \mathrm{mg} /$ day and has been studied only in patients receiving aspirin. ' In the cardiac catheterization laboratory, physicians provide an intravenous bolus dose of $0.75 \mathrm{mg} / \mathrm{kg}$ followed by an infusion of $1.75 \mathrm{mg} / \mathrm{kg} / \mathrm{hr}$ for the duration of the PCI. After 4 hours, an additional intravenous infusion may be given at a rate of $0.2 \mathrm{mg} / \mathrm{kg} / \mathrm{hr}$ for up to 20 hours.

The rate of major hemorrhage associated with bivalirudin is $4 \%$. There is no way to neutralize bivalirudin; however, in patients with normal renal function the half-life is 25 minutes. The dosage is decreased in patients with reduced GFR or elevated serum creatinine. Because bivalirudin is a small peptide, it does not elicit an immune response, thus no antihirudin-like antibodies to bivalirudin have been described.

\section{Dabigatran, the Oral Direct Thrombin Inhibitor}

Dabigatran etexilate (Pradaxa ${ }^{\circledR}$; Boehringer Ingelheim, Ingelheim, Germany) is an oral pro-drug that converts upon digestion to active dabigatran, a DTI that binds both free- and clot-bound thrombin. ${ }^{10}$ Dabigatran's efficacy and safety appear to match those of LMWH and Coumadin, and it has no interaction with food. ${ }^{11} \mathrm{It}$ is cleared by the kidneys, has a half-life of 12-17 hours, and is not metabolized by liver cytochrome enzymes. Dabigatran causes no liver toxicity. In the spring of 2009, dabigatran was cleared by agencies in Canada and northern Europe at $150 \mathrm{mg}$ once a day for venous thromboembolic (VTE) disease prophylaxis following total knee replacement or total hip replacement surgery. ${ }^{12}$ The US FDA cleared dabigatran for prevention of ischemic stroke in patients with atrial fibrillation in the fall of 2010 , making dabigatran the first oral anticoagulant to be cleared since Coumadin in 1954.

Dabigatran caused dyspepsia in $11 \%$ of clinical trial subjects. Its anticoagulant effect is immediate, but a single missed dose may expose the patient to renewed thrombosis risk. In case of overdose associated with bleeding, renal dialysis is the only means for reducing plasma levels, as there is no current medical means for neutralizing its effect. The question of laboratory monitoring in pregnant patients, the elderly, morbidly obese or underweight adults, and patients with renal dysfunction was not addressed during dabigatran 
clinical trials, but has become an initiative since the drug was released.

\section{Measuring Direct Thrombin Inhibitor Therapy}

All three DTIs prolong the PT, PTT, thrombin clotting time (TCT), and the activated clotting time (ACT). For nonsurgical therapy, argatroban and bivalirudin may be assayed using the PTT. The target therapeutic range is 1.5-3.0 times the mean of the laboratory-established reference interval (RI). Blood is collected 4 hours after the initiation of intravenous therapy for bivalirudin or 2 hours after starting argatroban, and the dosage is adjusted to achieve a PTT in the therapeutic range. Though generally available, the PTT is not specifically cleared for DTI monitoring, and there exist considerable sensitivity variations among reagent formulations and lots. However, because the duration of argatroban and bivalirudin therapy is relatively short, no attempt is being made to standardize the PTT for DTI monitoring.

The ACT may be used to assess argatroban or bivalirudin during PCI or coronary artery bypass graft surgery. ${ }^{13}$ During these procedures, the target ACT is 320-400 seconds (RI 90-175 seconds).

PTT reagents are not calibrated specifically to monitor argatroban or bivalirudin. Further, in instances in which the baseline PTT is prolonged by lupus anticoagulant, specific inhibitors, or factor deficiencies, the ecarin clotting time (ECT) is an attractive alternative for monitoring DTI therapy, including dabigatran therapy. Ecarin (ecarinase; Pentapharm, Basel, Switzerland) is an enzyme extracted from Echis carinatus venom that converts prothrombin to the intermediate meizothrombin, which converts fibrinogen to fibrin. ${ }^{14}$ DTIs bind meizothrombin and generate a linear, dosedependent prolongation of the ECT. Aside from DTIs, the ECT is prolonged only by abnormally low prothrombin and fibrinogen activity. Diagnostica Stago Inc has developed both a clot-based and a chromogenic ecarin assay, however both await FDA clearance.

Dabigatran, though it requires no regular monitoring, represents a special case compared to argatroban and bivalirudin. Its half-life of $12-17$ hours and its indication for long-term therapy make it more frequently implicated in hemorrhage or thrombosis. ${ }^{15}$ Consequently, dabigatran should be assayed in all the same circumstances as LMWH, as listed in Monitoring the Anti-Xa Anticoagulants. Further, it may be necessary to check for dabigatran compliance or to identify dabigatran in emergent hemorrhage.

Attempts to assay dabigatran using traditional coagulation tests have revealed that the PT is non-linear at therapeutic levels and that PTT reagents vary widely in dabigatran sensitivity. ${ }^{16}$ The TCT is linear but too sensitive. The TCT may be used semiquantitatively; a normal TCT indicates dabigatran absence, a TCT that exceeds 100 seconds may imply an overdose or renal retention. Though rarely used, the reptilase time assay is insensitive to dabigatran, thus a prolonged and uncorrectable PT, PTT, and TCT associated with a normal reptilase time result may indicate dabigatran.

Dabigatran may be assayed using the ECT or the PiCT assay described in the accompanying article, Monitoring the Anti-Xa Anticoagulants. BIOPHEN's Hemoclot ${ }^{\circledR}$ thrombin inhibitor assay, also known as the dilute TCT, is a simple procedure in which test plasma is mixed with normal plasma and the mixture is assayed using TCT reagent. This assay is linear and stable. Finally, the Helena point of care Abrazo ${ }^{\circledR}$ DTM provides near-patient testing based on the ECT. Regrettably, all current and proposed assays for dabigatran, and for the new oral anti-Xa anticoagulants rivaroxaban and apixaban are currently stalled at the FDA awaiting clearance. Meanwhile, laboratory operators are making do with the PTT, anti-Xa and the TCT. Given the efficacy, safety, and convenience of the new oral anticoagulants, these drugs and their assays may soon be made available worldwide.

\section{REFERENCES:}

1. Samama MM, Gerotziafas GT. Newer anticoagulants in 2009. J Thromb Thrombolysis 2010;29:92-104.

2. Guyatt GH, Akl EA, Crowther M, et al. Antithrombotic Therapy and Prevention of Thrombosis, 9th ed: American College of Chest Physicians Evidence-Based Clinical Practice Guidelines College of Chest Physicians Antithrombotic Therapy and Prevention of Thrombosis Panel Chest 2012;141(Suppl):7S-47S.

3. Yeh RW, Jang IK: Argatroban: update. Am Heart J 2006;151:1131-8.

4. Shantsila E, Lip GY, Chong BH. Heparin-induced thrombocytopenia. A contemporary clinical approach to diagnosis and management. Chest 2009;135:1651-64.

5. Linkins LA, Dans AL, Moores LK, et al. Treatment and prevention of heparin-induced thrombocytopenia: antithrombotic therapy and prevention of thrombosis, 9th ed: American 
College of Chest Physicians evidence-based clinical practice guidelines. Chest 2012;141(suppl):495-530S.

6. Fischer KG, Liebe V, Hudek R, et al. Anti-hirudin antibodies alter pharmacokinetics and pharmacodynamics of recombinant hirudin. Thromb Haemost 2003;89:973-82.

7. Cardenas GA, Deitcher SR: Risk of anaphylaxis after reexposure to intravenous lepirudin in patients with current or past heparin-induced thrombocytopenia. Mayo Clin Proc 2005;80:491-3.

8. Schulman S, Spencer FA. Antithrombotic drugs in coronary artery disease: risk benefit ratio and bleeding. J Thromb Haemost 2010;8:641-50.

9. Curran MP. Bivalirudin: in patients with ST-segment elevation myocardial infarction. Drugs 2010;70:909-18.

10. Wittkowsky AK. New oral anticoagulants: a practical guide for clinicians. J Thromb Thrombolysis 2010;29:182-91.

11. Connolly SJ, Ezekowitz MD, Yusuf S, et al. Dabigatran versus warfarin in patients with atrial fibrillation. NEJM 2009;361:1139-51.
12. Shulman S, Kearon C, Kakkar AK. Dabigatran versus warfarin in the treatment of acute venous thromboembolism. NEJM 2009;361:2342-52.

13. Chia S, Van Cott EM, Raffel OC, et al. Comparison of activated clotting times obtained using Hemochron and Medtronic analyzers in patients receiving anti-thrombin therapy during cardiac catheterization. Thromb Haemost 2009;101:535-40.

14. Choi TS, Khan AI, Greilich PE, et al: Modified plasma-based ecarin clotting time assay for monitoring of recombinant hirudin during cardiac surgery. Am J Clin Pathol 2006;125:290-5.

15. Uchino K, Hernandez AV. Dabigatran association with higher risk of acute coronary events: meta-analysis of noninferiority randomized controlled trials. Arch Intern Med 2012;172: 397-402.

16. Curvers J, van de Kerkhof D, Stroobants AK, et al. Measuring direct thrombin inhibitors with routine and dedicated coagulation assays. Am J Clin Pathol 2012;138:551-8.

\section{An outstanding webinar series by laboratory experts!}
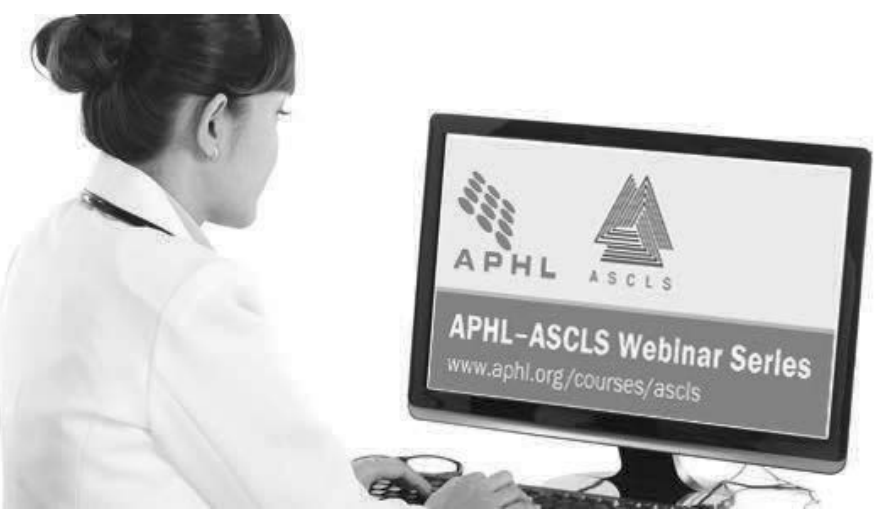

ASCLS members - register with special discount code for a reduced site rate!

Go to www.ascls.org/webinars for details.

Access to live and archived sessions with each purchase. Learn at your convenience! 